\title{
The Impact of Rehabilitation Interventions on Quality of Life in Indi- viduals with Amyotrophic Lateral Sclerosis: A Systematic Review
}

\author{
Ammarah Y Soofi*, Michelle E Kho, Vanina Dal Bello-Haas and Lori Letts \\ School of Rehabilitation Sciences, McMaster University, Canada

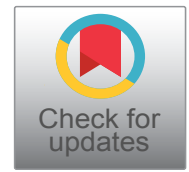

*Corresponding author: Ammarah Y Soofi, School of Rehabilitation Sciences, McMaster University, IAHS Rm. 402, 1400 Main Street West, Hamilton, Ontario L8S 1C7, Canada, E-mail: soofiay@mcmaster.ca

\begin{abstract}
Background: Many factors can affect a person's outlook on his/her life; rehabilitation interventions vary greatly and little is known about the effect of rehabilitation to maintain and/ or enhance Quality of Life (QoL) in people with Amyotrophic Lateral Sclerosis (ALS).
\end{abstract}

Objective: This systematic review aimed to determine the effectiveness of interventions within the scope of rehabilitation including Physio-Therapy (PT), Occupational Therapy (OT) and Speech and Language Pathology (SLP) on QoL in people with ALS.

Methods: A comprehensive search of databases was performed. Inclusion criteria were adults diagnosed with ALS who received rehabilitation interventions from the PT, OT, and/or SLP professions reporting QoL outcomes measured by the Short-Form 36 Questionnaire or ALS Assessment Questionnaire-40 (ALSAQ-40). We included quantitative and/or mixed-methods studies. We assessed quality of reporting and appraised risk of bias using appropriate tools for Randomized Controlled Trials (RCTs) and non-RCTs.

Results: We conducted a narrative synthesis of interventions because available data were insufficient for meta-analysis. Six studies enrolling 460 ALS patients met inclusion criteria: three RCTs, two cohort studies, and one cross-sectional study. Interventions included exercise, communication devices, and multidisciplinary care. Six studies reported SF-36 and one reported ALSAQ-40. The studies found that people using rehabilitation interventions have a higher QoL prior to receiving interventions.

Conclusion: To date little evidence is available quantifying positive and sustained effects of interventions from PT, OT, and SLP on QoL measured by the SF-36 or ALSAQ-40 in the ALS population. While interventions by PT, OT, and SLP have the potential to play an important role in delivering appropriate management of ALS to improve QoL, further research is needed.

\section{Keywords}

Amyotrophic Lateral Sclerosis (ALS), Physio-Therapy (PT), Occupational Therapy (OT), Speech and Language Pathology (SLP), Quality of Life (QoL), Rehabilitation

\section{Background}

Amyotrophic Lateral Sclerosis (ALS) is a degenerative, disabling and fatal motor neuron disease affecting 450,000 adults worldwide [1]. The prognosis of ALS is affected by age at disease onset, rate of progression, and presence of bulbar signs early in the disease [2,3]. Currently there is no cure for ALS and the cause of the disease remains unknown. Therefore, treatment is based on symptom management to optimize lifespan and maximize QoL $[4,5]$.

Individuals with ALS experience muscle weakness due to the progressive degeneration of motor neurons in the spinal cord, brainstem, and cerebral hemispheres [6]. This ultimately results in activity limitations and participation restrictions for these individuals. About 50\% of people experience initial signs and symptoms in the upper extremities, whereas for $40 \%$ of individuals the onset occurs in the lower extremities [7]. In the remaining $10 \%$, onset may be observed in areas of the bulbar region showing symptoms of changes in voice and speech, poor articulation, a decrease in range of pitch and loudness of voice, spasms in muscles of the jaw, face, voice box, throat and tongue [8].

As the disease progression can occur rapidly, it is essential that people with ALS have quick access to services from a range of health professionals that are 
skilled and effective in the management of the physical and psychosocial developments that occur with this condition $[9,10]$. Several interventions from Physio-Therapists (PTs), Occupational Therapists (OTs), and Speech and Language Pathologists (SLPs) can directly target functional impairments, physical activity, and address patients' limitations with regard to participation in social, home, work, leisure, and community roles [6]. The nature of ALS can significantly affect a person's feelings of hope and self-worth. Additionally, one's personal activities, fulfillment of life goals, and continuing employment are also affected [11]. Changes in self-esteem, loss of independence, and psychosocial stress all play important roles in an individual's experiences of QoL throughout the disease process [2]. To maintain independence and maximize QoL, many individuals seek the services of rehabilitation professionals for the purposes of "advice, education, movement rehabilitation, speech/swallowing rehabilitation, environmental modification, workplace adaptation, and the provision of assistive devices" [6] for environmental control and to assist with communication.

Rehabilitation is a process aimed at enabling people with disabilities to attain and maintain the best "physical, intellectual, psychological and social functional levels" possible by offering the tools necessary to manage independence and control of their life [12]. Rehabilitation professionals use their knowledge and expertise to assist individuals with ALS to understand the disease progression, control symptoms, manage expectations, address communication issues, identify and address end of life issues, fulfill equipment needs, provide counselling and support, and advise on social assistance [13]. This broad range of tasks fulfilled by rehabilitation professionals collaboratively can maintain and/or enhance QoL of the person with ALS. QoL has been defined as "the extent to which our hopes and ambitions are matched by experience" [11]; thus it refers to a person's satisfaction with the way his/her life is progressing, despite the diagnosis of a disease such as ALS, and with the care measures being taken [11]. Although there are many other factors that can affect a person's outlook on his/her life after diagnosis, this study focuses specifically on the effect of rehabilitation on QoL. This review specifically addresses interventions from PT, OT, and SLP, and multidisciplinary care that include one or all of these therapies to examine the impact on QoL in people with ALS. Our research question was: In individuals with ALS, what interventions within the scope of rehabilitation are effective for improving QoL?

\section{Methods}

\section{Search strategy}

The search was conducted by author AYS in March 2015 with the following restrictions: English articles only, dated from 1980 to March 2015, and studies with human participants. We chose 1980 because Mikulic, DeLisa, and Miller [14] proposed the idea of using rehabilitation for people with ALS in 1979. The search was conducted using the PICO approach (Appendix 1) in consultation with a health sciences librarian and included the following databases: MEDLINE; CINAHL; PsycINFO; The Physiotherapy Evidence Database (PEDro); Occupational Therapy Systematic Evaluation of Evidence (OT seeker); AMED; EMBASE; OVID Health Star; Global Health; and LILACS EN. The main search terms included amyotrophic lateral sclerosis, motor neurone disease, motor neuron disease, physiotherapy, physical therapy, occupational therapy, speech language pathology, rehabilitation, therapy, and quality of life (Appendix 1).

\section{Types of studies}

The authors searched for all quantitative study designs that included interventions delivered by PT, OT and/or SLP for people with ALS. Inclusion criteria: People with a diagnosis of ALS (duration and stage of the disease did not have to be reported); rehabilitation intervention(s) (Appendix 2) delivered by at least one of the disciplines of PT, OT, SLP, or delivered by another person or discipline and assigned by one of these rehabilitation professionals; QoL outcomes (see below) and quantitative and/or mixed-method designs (i.e. Randomized Control Trials (RCTs), cohort, cross-sectional, and case-control studies). We included studies if part of a sample included people with ALS and met our other inclusion criteria.

\section{Outcome measures}

The primary outcome of this study was Quality of Life (QoL) as measured by the 36 item Short-Form Survey (SF-36) questionnaire [15] or the Amyotrophic Lateral Sclerosis Assessment Questionnaire (ALSAQ-40) [16]. In the SF-36 questionnaire [15], there are eight sections; vitality, physical functioning, bodily pain, general health perceptions, physical role functioning, emotional role functioning, social role functioning, and mental health. In this measure higher scores reflect higher health-related QoL (i.e., a score of zero is equivalent to poor QoL and a score of 100 is equivalent to excellent QoL).

The ASLAQ-40 has 40 questions specific to the ALS population with 5 discrete scales: Physical mobility, activities of daily living and independence, eating and drinking, communication, and emotional reactions. Individuals with ALS are asked questions about the difficulties experienced during the last two weeks (e.g. I have fallen over while walking). The responses are collected by selecting one of five options (Likert scale): Never/rarely/sometimes/often/always or cannot do at all [16]. The responses are reported and collected where a higher number of responses in the "never/rarely/sometimes" section indicates a better QoL and, subsequently, responses in the "often/always or cannot do at all" correlates to a lower QoL. Thus, in contrast to the SF-36, 


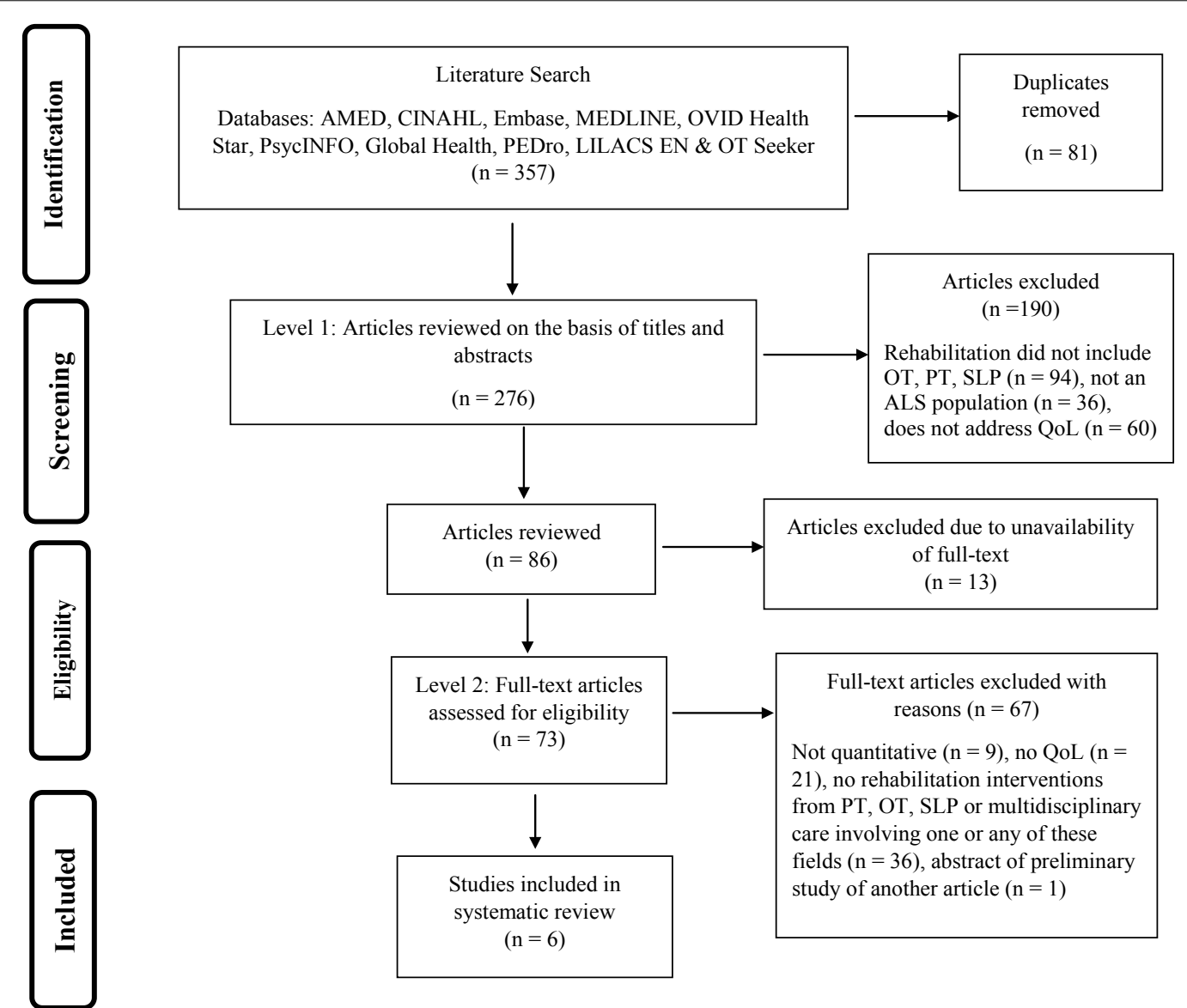

Figure 1: PRISMA flow diagram for study selection in Systematic Review.

higher scores on the ALSAQ-40 indicate lower QoL.

\section{Data extraction and quality appraisal}

Selection of studies: Two review authors (AYS and LL) independently screened titles and abstracts of all citations. All potentially relevant articles selected by either author were obtained as full-text articles, assessed for inclusion and exclusion criteria and documented reasons for exclusion. Where there were differences between reviewers, a discussion occurred to arrive at consensus. Kappa was calculated to determine the level of inter-rater agreement [17]. Once the studies were identified, the primary author (AYS) used a data extraction form (Appendix 2) to describe the study characteristics, study participants and conclusions. Data extracted provided information about the study's purpose, ethical approval, methodology, outcome measures and results. All findings were compiled in a single chart.

Quality appraisal and assessment of risk of bias: Two review authors (AYS and LL) independently evaluated the quality of the reporting of RCTs using the CONSORT Checklist [18] and risk of bias using the Cochrane Risk of bias tool [19]. We assessed risk of bias as "high", "low" or "unclear". The STROBE Statement [20] and the New-Castle Ottawa scale [21] were used to assess quality of reporting and risk of bias in the non-RCT studies. In the case of disagreement between the two authors, each reassessed the studies and agreement was reached through discussion.
Statistical analysis: We planned to conduct a meta-analysis by QoL outcome if the studies were clinically (e.g. similar intervention) and statistically (e.g. all SF-36 results reported by the mean of each group) homogeneous [22]. The authors contacted included studies' authors for additional data if required.

\section{Results}

After removing duplicates, the search yielded 276 articles, and 7 citations (Figure 1); however, one citation was an abstract [23] and it was removed as it was a preliminary data report of another study [24], resulting in a total of 6 unique studies. Three studies were RCTs [24-26], two were cohort studies $[27,28]$ and one was a cross-sectional study [29]. Five studies reported SF-36 [24,25,27-29] and one study reported ALSAQ-40 [26]. There were no disagreements between the authors for included studies; using Cohen's Kappa [16], the level of agreement was calculated as 1 , representing perfect agreement.

Following contact with study authors for additional data, meta-analyses could not be conducted because the studies were too heterogeneous. Thus, we conducted a narrative synthesis. Table 1 presents a summary of all included studies. Table 2 presents the evaluation of the quality of the studies based on the CONSORT and STROBE criteria (Appendix 2), where a higher percentage in total score is interpreted as higher quality of reporting. Table 3 presents the results of the risk of bias assessments. 
Table 1: Details of Included Studies.

\begin{tabular}{|c|c|c|c|c|c|c|}
\hline $\begin{array}{l}\text { Author \& } \\
\text { publication year, } \\
\text { study design, and } \\
\text { total sample size } \\
\text { (evaluated for } \\
\text { QoL/enrolled) }\end{array}$ & $\begin{array}{l}\text { Purpose of the } \\
\text { study }\end{array}$ & $\begin{array}{l}\text { Intervention } \\
\text { (evaluated/ } \\
\text { enrolled) }\end{array}$ & $\begin{array}{l}\text { Control } \\
\text { (evaluated/ } \\
\text { enrolled) }\end{array}$ & $\begin{array}{l}\text { Outcome } \\
\text { measures } \\
\text { (QoL } \\
\text { measure in } \\
\text { bold) }\end{array}$ & $\begin{array}{l}\text { Overall study } \\
\text { results }\end{array}$ & QoL results \\
\hline $\begin{array}{l}\text { Drory, et al., } 2001 \\
\text { Randomized } \\
\text { Controlled Trial; } \\
\mathrm{N}=14 / 25 \text { (males) } \\
\text { Moderate Daily } \\
\text { Exercise (DE) vs. } \\
\text { No exercise or } \\
\text { Physical Activity } \\
\text { other than daily } \\
\text { life activities (PA) }\end{array}$ & $\begin{array}{l}\text { To determine } \\
\text { the effect } \\
\text { moderate } \\
\text { exercise under } \\
\text { professional } \\
\text { guidance have } \\
\text { on motor deficit, } \\
\text { disability, } \\
\text { fatigue, MSK } \\
\text { pain and QoL }\end{array}$ & $\begin{array}{l}\text { Daily exercise } N \\
=8 / 14 \text { (males) }\end{array}$ & $\begin{array}{l}\text { Daily } \\
\text { activities } \\
\mathrm{N}=6 / 11 \\
\text { (males) }\end{array}$ & $\begin{array}{l}\text { MMT, } \\
\text { Ashworth } \\
\text { spasticity } \\
\text { scale, } \\
\text { ALSFRS, } \\
\text { FSS, VAS, } \\
\text { SF-36 }\end{array}$ & $\begin{array}{l}\text { QoL } \\
\text { deteriorated } \\
\text { slightly with a } \\
\text { non-significant } \\
\text { trend towards } \\
\text { the control } \\
\text { group }\end{array}$ & $\begin{array}{l}\text { Mean at baseline: } \\
\text { PA - } 84.1 \\
\text { DE }-82.9 \\
\text { Mean at } 3 \text { months: } \\
\text { PA - } 83.0 \\
D E-80.4 \\
\text { Mean at } 6 \text { months: } \\
\text { PA - } 81.2 \\
\text { DE - } 72.7\end{array}$ \\
\hline $\begin{array}{l}\text { Dal Bello-Haas, } \\
\text { et al., } 2007 \\
\text { Randomized } \\
\text { Control Trial; } \\
\mathrm{N}=27 \\
\text { Resistance } \\
\text { Exercise (RE) } \\
\text { vs. Stretching } \\
\text { Exercise (SE) }\end{array}$ & $\begin{array}{l}\text { To determine } \\
\text { the effects } \\
\text { of resistance } \\
\text { exercise on } \\
\text { function, } \\
\text { fatigue, and } \\
\text { QoL }\end{array}$ & $\begin{array}{l}\text { Resistance } \\
\text { exercise = } 13\end{array}$ & $\begin{array}{l}\text { Stretching } \\
\text { group }=14\end{array}$ & $\begin{array}{l}\text { ALSFRS, } \\
\text { FSS, SF- } \\
\text { 36, Muscle } \\
\text { strength } \\
\text { (MVIC) }\end{array}$ & $\begin{array}{l}\text { No adverse } \\
\text { effects occurred } \\
\text { with relation } \\
\text { to intervention } \\
\text { and decline in } \\
\text { leg strength } \\
\text { was lower in } \\
\text { resistance } \\
\text { exercise group }\end{array}$ & $\begin{array}{l}3 \text { months: } \\
\text { No difference between } \\
\text { groups (SE and RE) } \\
6 \text { months: RE significantly } \\
\text { higher than SE } \\
t=2.58 \\
\text { df }=16 \\
p=0.02\end{array}$ \\
\hline $\begin{array}{l}\text { Creemers, et al., } \\
2013 \\
\text { Cluster } \\
\text { Randomized } \\
\text { Controlled Trial; N } \\
=298 \\
\text { Multidisciplinary } \\
\text { ALS Care with } \\
\text { Case management } \\
\text { (MDC+CS) } \\
\text { vs. Usual } \\
\text { Multidisciplinary } \\
\text { ALS Care (MDC) }\end{array}$ & $\begin{array}{l}\text { To determine } \\
\text { the effect } \\
\text { of case } \\
\text { management on } \\
\text { QoL, caregiver } \\
\text { strain, and } \\
\text { perceived } \\
\text { Quality of } \\
\text { Care (QOC) in } \\
\text { patients with } \\
\text { ALS and their } \\
\text { carers }\end{array}$ & $\begin{array}{l}\text { Case } \\
\text { management } \\
\text { and usual care } \\
\text { ALS patients } N \\
=71 \text {, Caregivers } \\
\mathrm{N}=66\end{array}$ & $\begin{array}{l}\text { Usual } \\
\text { care ALS } \\
\text { patients } \\
\mathrm{N}=61 \\
\text { Caregivers } \\
\mathrm{N}=60\end{array}$ & $\begin{array}{l}\text { ALSFRS-R, } \\
\text { ALSAQ-40, } \\
\text { CSI, QOC }\end{array}$ & $\begin{array}{l}\text { Introducing } \\
\text { case } \\
\text { management } \\
\text { for people with } \\
\text { ALS showed no } \\
\text { benefit in QoL } \\
\text { scores. }\end{array}$ & $\begin{array}{l}\text { Mean (SD), } n \\
\text { (Emotional Functioning)- } \\
\text { Baseline MDC + CS: } 21.3 \\
\text { (18.2), } 70 \\
\text { Baseline MDC: } 19.4 \\
\text { (17.0), } 61 \\
\text { p-value: } 0.524 \\
\\
4 \text { months MDC + CS: } \\
19.8 \text { (14.6), } 43 \\
4 \text { months MDC: } 19.4 \\
\text { (16.6), } 39 \\
\text { p-value: } 0.35 \\
\text { 8 months MDC + CS: } \\
21.5 \text { (13.5), } 28 \\
8 \text { months MDC: } 20.0 \\
\text { (16.1), } 22 \\
\text { p-value: } 0.33 \\
\text { 12 months MDC + CS: } \\
22.8 \text { (16.4), } 30 \\
12 \text { months MDC: } 19.1 \\
\text { (14.7), } 27 \\
\text { p-value: } 0.68 \\
\end{array}$ \\
\hline
\end{tabular}




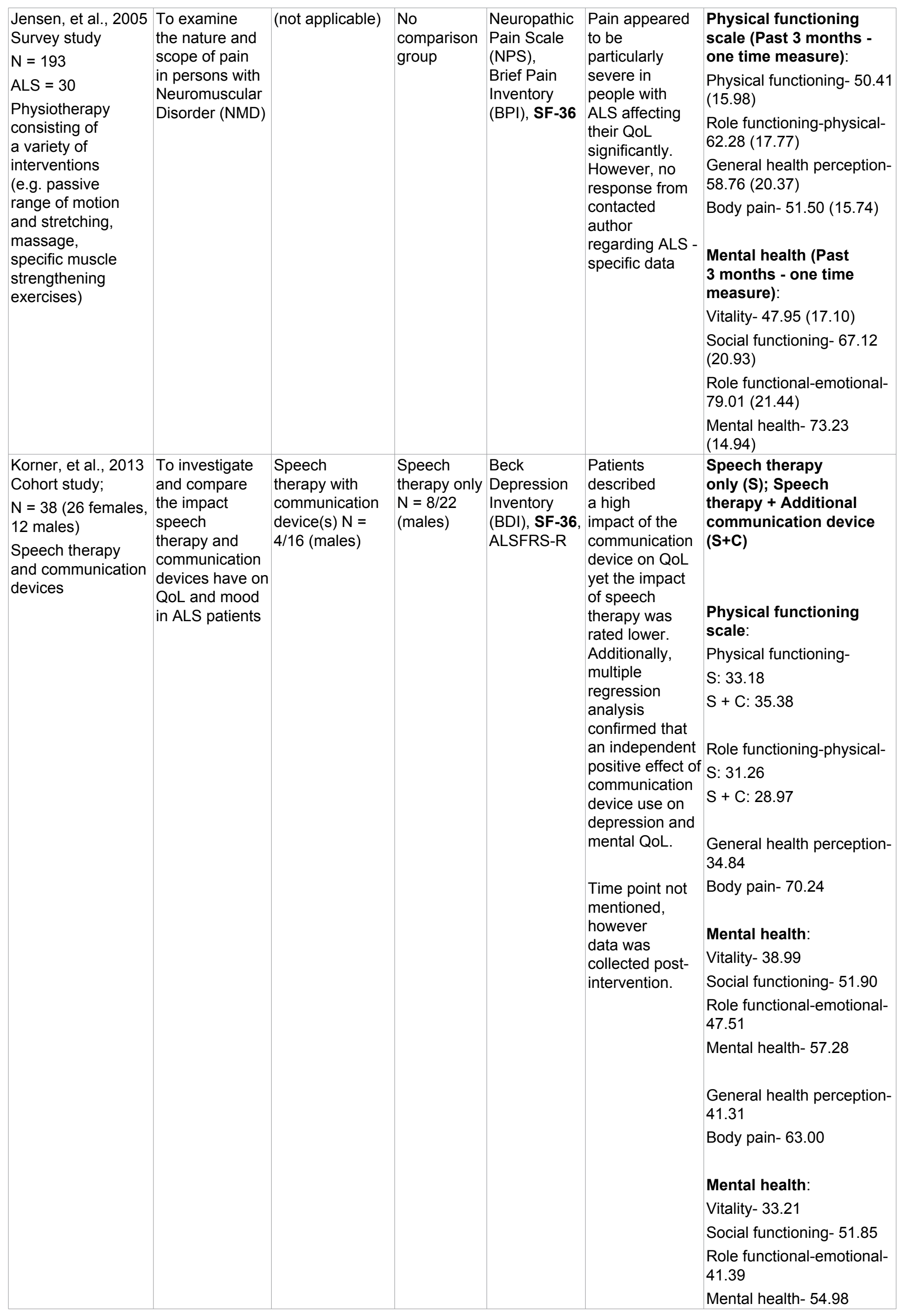




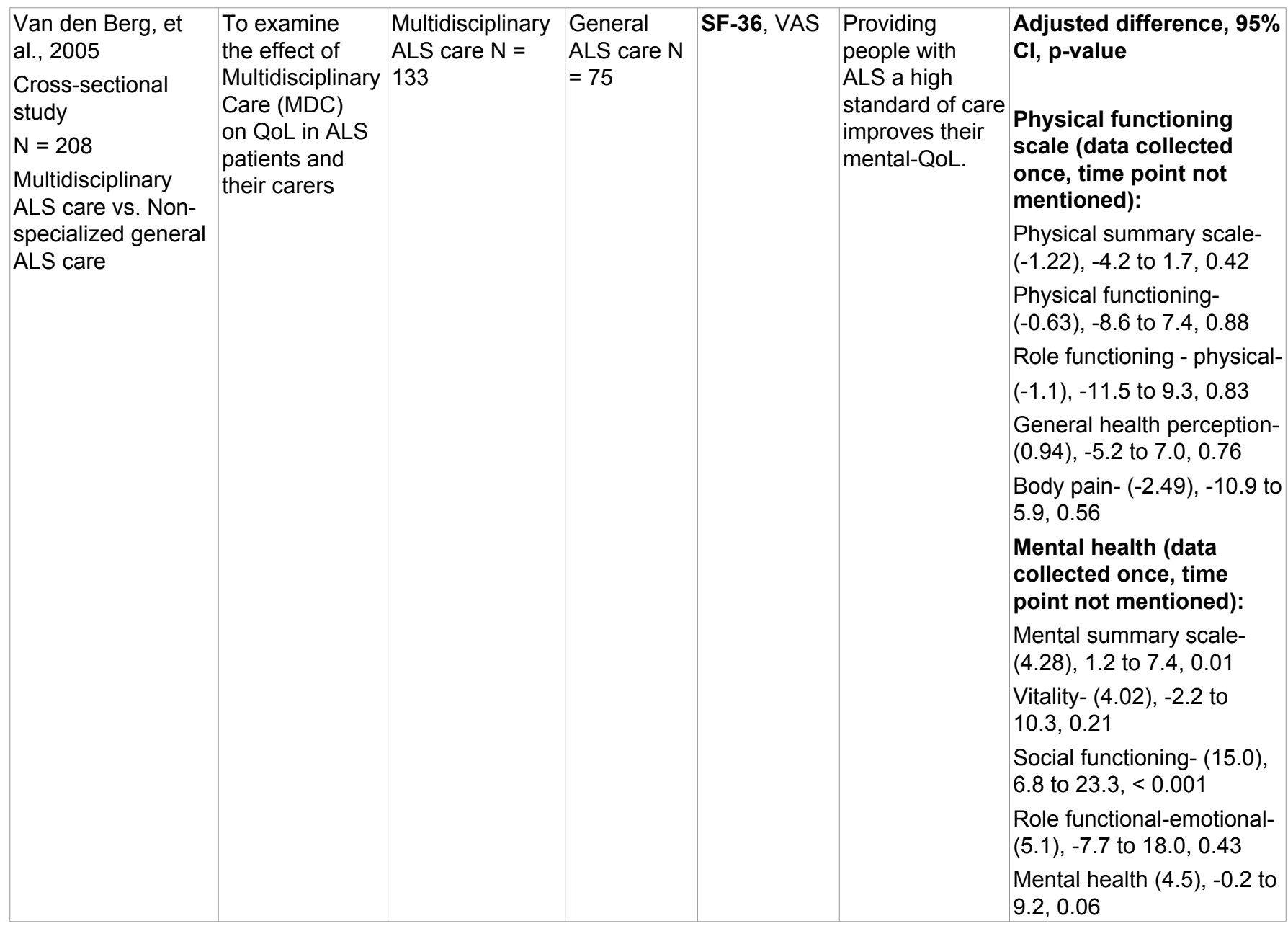

Abbreviations: MMT: Manual Muscle Testing; ALSFRS: Amyotrophic Lateral Sclerosis Functional Rating Scale; FSS: Fatigue Severity Scale; VAS: Visual Analogue Scale; SF-36: Short Form-36; Muscle strength MVIC: Maximum Voluntary Isometric Contraction; ALSAQ-40: Amyotrophic Lateral Sclerosis Assessment Questionnaire-40; CSI: Caregiver Strain Index; QOC: Quality of Care; NPS: Neuropathic Pain Scale; BPI: Brief Pain Inventory; BDI: Beck Depression Inventory; MDC: Multi-Disciplinary Care; CM: Case Management; RE: Resistance Exercise; SE: Stretching Exercise; DE: Moderate Daily Exercise (DE); PA: no exercise or Physical Activity other than daily life activities.

Table 2: Study reporting quality of the included studies based on CONSORT (Studies 1-3) and STROBE criteria (Studies 4-6).

\begin{tabular}{|c|c|c|c|c|c|c|c|c|c|c|}
\hline \multicolumn{2}{|c|}{$\begin{array}{l}\text { Author and year of } \\
\text { publication }\end{array}$} & \multirow{2}{*}{$\begin{array}{l}\text { Title \& } \\
\text { Abstract } \\
1\end{array}$} & \multirow{2}{*}{$\begin{array}{l}\text { Introduction } \\
1\end{array}$} & \multirow{2}{*}{$\begin{array}{l}\text { Methods } \\
10\end{array}$} & \multirow{2}{*}{$\begin{array}{l}\text { Results } \\
7\end{array}$} & \multirow{2}{*}{\begin{tabular}{|l} 
Discussion \\
3 \\
\end{tabular}} & \multirow{2}{*}{$\begin{array}{l}\text { Other } \\
\text { information } \\
3 \\
\end{array}$} & \multirow[t]{2}{*}{$\begin{array}{l}\text { Score } \\
\text { obtained }\end{array}$} & \multirow[t]{2}{*}{$\begin{array}{l}\text { Total } \\
\text { score }\end{array}$} & \multirow[t]{2}{*}{$\begin{array}{l}\% \text { of Total } \\
\text { score }\end{array}$} \\
\hline & CONSORT & & & & & & & & & \\
\hline 1. & Drory, et al., 2001 & 0.5 & 1 & 7 & 6 & 3 & 2 & 19.5 & 25 & $78 \%$ \\
\hline 2. & $\begin{array}{l}\text { Dal Bello-Haas, et } \\
\text { al., } 2007\end{array}$ & 1 & 1 & 8 & 6 & 3 & 3 & 22 & 25 & $88 \%$ \\
\hline \multirow[t]{2}{*}{3.} & $\begin{array}{l}\text { Creemers, et al., } \\
2013\end{array}$ & 1 & 1 & 8 & 7 & 2 & 3 & 22 & 25 & $88 \%$ \\
\hline & STROBE & 1 & 2 & 9 & 5 & 4 & 1 & & & \\
\hline 4. & $\begin{array}{l}\text { Jensen, et al., } \\
2005\end{array}$ & 1 & 2 & 6 & 3 & 3.5 & 1 & 16.5 & 22 & $75 \%$ \\
\hline 5. & Korner, et al., 2013 & 0.5 & 2 & 7 & 3 & 3 & 0 & 15.5 & 22 & $70 \%$ \\
\hline 6. & $\begin{array}{l}\text { Van den Berg, et } \\
\text { al., } 2005\end{array}$ & 1 & 2 & 7 & 4 & 4 & 1 & 19 & 22 & $86 \%$ \\
\hline
\end{tabular}

\section{Description of studies and effect of rehabilitation interventions on QoL using SF-36 and ALSAQ-40}

SF-36: Of the five studies reporting SF-36 $[24,25,27-$ 29], three were RCTs, and 2 were non-RCTs. Two RCTs which focused on exercise enrolled 25 and 27 ALS patients respectively [24,25]. Drory, et al. [25] compared people that performed exercise to those whose physical activities only included Activities of Daily Living (ADLs).
The exercise program targeted muscle endurance and resistance against a modest load for people with ALS [25]. This study had no statistically significant differences in QoL between the two groups (Control Group Mean $=81.2$, Exercise Group Mean = 72.7). Dal Bello-Haas, et al. [24] determined the effects of resistance exercise on function, fatigue, and QoL, the study found that at 6 months, the resistance exercise group presented a significantly higher QoL (SF-36) score than the control group 
Table 3: Results of Risk of bias evaluation.

\begin{tabular}{|c|c|c|c|c|c|c|}
\hline $\begin{array}{l}\text { Author \& } \\
\text { Publication year }\end{array}$ & $\begin{array}{l}\text { Random sequence } \\
\text { generation } \\
\text { (selection bias) }\end{array}$ & $\begin{array}{l}\text { Allocation } \\
\text { concealment } \\
\text { (selection bias) }\end{array}$ & $\begin{array}{l}\text { Blinding } \\
\text { (performance } \\
\text { bias and } \\
\text { detection } \\
\text { bias) All } \\
\text { outcomes }\end{array}$ & $\begin{array}{l}\text { Incomplete } \\
\text { outcome data } \\
\text { (attrition bias) } \\
\text { All outcomes }\end{array}$ & $\begin{array}{l}\text { Selective } \\
\text { reporting } \\
\text { (reporting bias) }\end{array}$ & $\begin{array}{l}\text { Overall risk of } \\
\text { bias }\end{array}$ \\
\hline $\begin{array}{l}\text { Drory, et al., } \\
2001\end{array}$ & $\begin{array}{l}\text { Low risk - The } \\
\text { institution statistician } \\
\text { performed the } \\
\text { randomization using } \\
\text { computer software }\end{array}$ & $\begin{array}{l}\text { Unclear risk - not } \\
\text { reported }\end{array}$ & $\begin{array}{l}\text { Unclear risk } \\
\text { - blinding not } \\
\text { reported }\end{array}$ & $\begin{array}{l}\text { High risk - Both } \\
\text { groups lost a } \\
\text { lot of people- } \\
\text { reasons for drop } \\
\text { outs not reported } \\
\text { - numbers are }\end{array}$ & $\begin{array}{l}\text { Low risk - All } \\
\text { outcomes } \\
\text { reported }\end{array}$ & Unclear risk \\
\hline $\begin{array}{l}\text { Dal Bello-Haas, } \\
\text { et al., } 2007\end{array}$ & $\begin{array}{l}\text { Low risk - random } \\
\text { sequence reported }\end{array}$ & $\begin{array}{l}\text { Low risk } \\
\text { - Opaque } \\
\text { envelopes with } \\
\text { group allocation } \\
\text { used; note that } \\
\text { participants were } \\
\text { not blind to group } \\
\text { allocation and } \\
\text { some revealed } \\
\text { their allocation } \\
\text { to therapist } \\
\text { collecting data }\end{array}$ & $\begin{array}{l}\text { Low risk - } \\
\text { Participants } \\
\text { completing } \\
\text { intervention } \\
\text { were } \\
\text { unblinded; } \\
\text { outcome } \\
\text { evaluators } \\
\text { were blinded } \\
\text { to group } \\
\text { allocation }\end{array}$ & $\begin{array}{l}\text { Low risk - } \\
\text { Participants } \\
\text { were lost to } \\
\text { follow up in both } \\
\text { groups- reasons } \\
\text { clearly stated } \\
\text { and missing data } \\
\text { were imputed for } \\
\text { analyses }\end{array}$ & $\begin{array}{l}\text { Low risk - All } \\
\text { outcomes } \\
\text { reported }\end{array}$ & Low risk \\
\hline $\begin{array}{l}\text { Creemers, et al., } \\
2013\end{array}$ & $\begin{array}{l}\text { Low risk - All ALS } \\
\text { teams were invited } \\
\text { to participate; teams } \\
\text { were matched }\end{array}$ & $\begin{array}{l}\text { Low risk - } \\
\text { Allocation } \\
\text { concealment was } \\
\text { at the cluster } \\
\text { level }\end{array}$ & $\begin{array}{l}\text { Low risk } \\
\text { - blinded } \\
\text { outcome } \\
\text { evaluators at } \\
\text { all follow-up } \\
\text { times }\end{array}$ & $\begin{array}{l}\text { Low risk - } \\
\text { Although they } \\
\text { lost quite a few } \\
\text { participants from } \\
\text { both groups, } \\
\text { the losses are } \\
\text { explained and } \\
\text { many were } \\
\text { because of death }\end{array}$ & $\begin{array}{l}\text { Low risk - All } \\
\text { outcomes } \\
\text { reported }\end{array}$ & Low risk \\
\hline $\begin{array}{l}\text { Jensen, et al., } \\
2005 \\
\text { Note: Data } \\
\text { presented does } \\
\text { not clearly } \\
\text { identify people } \\
\text { with ALS }\end{array}$ & $\begin{array}{l}\text { Low risk - } \\
\text { People with } \\
\text { neurodegenerative } \\
\text { conditions were } \\
\text { allowed to complete } \\
\text { the survey }\end{array}$ & $\begin{array}{l}\text { Low risk - Limited } \\
\text { restrictions } \\
\text { on who could } \\
\text { participate on the } \\
\text { survey }\end{array}$ & $\begin{array}{l}\text { Low risk - No } \\
\text { outcomes } \\
\text { based on } \\
\text { performance, } \\
\text { all self-report } \\
\text { measures }\end{array}$ & $\begin{array}{l}\text { Low risk - } \\
\text { Onetime survey } \\
\text { therefore no } \\
\text { attrition }\end{array}$ & $\begin{array}{l}\text { Low risk - data } \\
\text { on all outcomes } \\
\text { reported }\end{array}$ & Low risk \\
\hline $\begin{array}{l}\text { Korner, et al., } \\
2013\end{array}$ & $\begin{array}{l}\text { Low risk - } \\
\text { Participant selection } \\
\text { mentioned }\end{array}$ & $\begin{array}{l}\text { Low risk - Groups } \\
\text { assigned based } \\
\text { on allocation }\end{array}$ & $\begin{array}{l}\text { Low risk - all } \\
\text { outcome } \\
\text { measures are } \\
\text { self-report }\end{array}$ & $\begin{array}{l}\text { Low risk - data } \\
\text { collected when } \\
\text { patients visited } \\
\text { the clinic post- } \\
\text { intervention }\end{array}$ & $\begin{array}{l}\text { Unclear risk - } \\
\text { Can't identify } \\
\text { dependent and } \\
\text { independent } \\
\text { variables in } \\
\text { regression }\end{array}$ & Low risk \\
\hline $\begin{array}{l}\text { Van den Berg, et } \\
\text { al., } 2005\end{array}$ & $\begin{array}{l}\text { Unclear risk - No } \\
\text { random selection } \\
\text { and not clear how } \\
\text { patients were } \\
\text { recruited or selected }\end{array}$ & $\begin{array}{l}\text { Unclear risk - } \\
\text { not clear how } \\
\text { allocations were } \\
\text { determined }\end{array}$ & $\begin{array}{l}\text { Low risk } \\
\text { - nurses } \\
\text { collecting } \\
\text { outcome } \\
\text { data blinded } \\
\text { to treatment } \\
\text { group }\end{array}$ & $\begin{array}{l}\text { Unclear risk - no } \\
\text { clear description } \\
\text { of dropouts nor } \\
\text { if patients were } \\
\text { followed over time }\end{array}$ & $\begin{array}{l}\text { Low risk - all } \\
\text { outcome } \\
\text { measures } \\
\text { reported }\end{array}$ & Unclear risk \\
\hline
\end{tabular}

(stretching exercise). The resistance exercise group was given a stretching exercise program with an individualized Upper (U/E) and Lower Extremity (L/E) resistance exercise program with moderate load and intensity. The control group received a program that consisted of daily $U / E$ and $L / E$ stretching exercises to be performed once a day. The studies were mainly limited by sample sizes, brief duration of intervention, and drop-outs thus affecting the statistical power of results. Of the two studies, [25] observed a non-significant decline in QoL towards the control group using the SF-36 (Table 1).
Interventions delivered by SLPs were addressed in one study [27]. Korner, et al. [27] conducted a cohort study of patients with ALS who received speech therapy alone or speech therapy combined with communication devices. The response rate was $100 \%$, and included 38 participants. This study found that speech therapy with communication devices positively affected QoL, however there were no significant between group differences on any component of the SF-36. Nevertheless, the study found that communication devices and speech therapy appeared to stabilize QoL in people with ALS. 
Jensen, et al. [28] conducted a survey on the nature of pain and pain treatments (including rehabilitation interventions such as muscle strengthening exercises) used for people with neuromuscular disease, including those with ALS. The response rate was 30\% from people with ALS and $86 \%$ response rate overall. Of 193 participants, 30 had ALS. Across all the neuromuscular conditions, physiotherapeutic approaches were reported to provide the greatest pain relief, however this did not show an impact on the QoL score measured using the SF-36 questionnaire. The results of this study were reported for the whole sample, limiting the ability to identify the importance of physiotherapy for pain management in people with ALS. However, the authors noted that people with ALS appeared to be the most sensitive to the effects of pain, thus potentially influencing QoL. Therefore, the study was unable to link the effectiveness of physiotherapy interventions for pain to improved QoL.

The cross-sectional study performed by Van den Berg, et al. [29] assessed the effect of multidisciplinary ALS care from a team delivered according to the Dutch consensus protocol for rehabilitation management in ALS compared to general care. The ALS team was led by a consultant in rehabilitation medicine and included a PT, an OT, an SLP, a dietician, or a social worker. This study found better SF-36 mental-QoL among people who received multidisciplinary care compared to those who only received general care; however this was not statistically significant in the multivariate analysis. Yet in the multivariate analysis performed by Van den Berg, et al. [29] to determine the factors that contributed to changes in mental-QoL, it remains unknown which factors contributed to improved mental-QoL. Despite the discussion sections of the studies reporting the positive influence of rehabilitation services to impact QoL, use of the SF-36 questionnaire typically resulted in non-statistically significant results comparing outcomes between groups.

ALSAQ-40: Creemers, et al. [26] conducted a cluster RCT of multidisciplinary interventions in 132 people with ALS to establish the effect of case management for patients and caregivers compared to usual multidisciplinary ALS care. The study reported no benefit of case management to improve QoL measured by the ALSAQ-40, unless people with ALS were struggling with using and/or accessing rehabilitation interventions or other care services. This study reported that during the intervention period, people with ALS reported one issue related to a rehabilitation intervention whereas other reasons for seeing a case manager were related to emotional support. Despite this noticeable trend among the participants, the emotional function scores on the ALSAQ-40 measure had no significant difference than the usual multidisciplinary ALS care group.

\section{Quality of the evidence}

We assessed the quality of reporting using the CON-
SORT statement, STROBE statement, and the Cochrane Risk of Bias Tool as appropriate to each study design. Five of the six included studies had overall reporting percentages above $75 \%$ (Table 2). The study that scored less than $75 \%$ [27] was due to unmet criteria from the results section (i.e. information was not provided regarding reasons for participants not participating at each stage, participant characteristics were incomplete, and additional details were missing to describe the study methods).

From the Cochrane Risk of bias assessments, the authors determined that the Drory, et al. [25] study was at unclear risk of bias, and that the Dal Bello-Haas, et al. [24] and Creemers, et al. [26] studies were at low risk of bias. In two studies $[25,26]$, there were no baseline differences between participant groups in regards to age and disease duration. In Drory, et al. [25], the institution statistician performed the randomization using computer software, Dal Bello-Haas, et al. [24] reported that opaque envelopes with group allocation was used, and the Creemers, et al. [26] reported that all ALS teams were invited to participate in the study then teams were matched based on three factors. In two studies [24,25], allocation concealment was not reported, however in the cluster RCT [26] allocation concealment was performed at the cluster level. Additionally, as it would be difficult to blind participants to the exercise intervention, Drory, et al. [25] did not blind the participants, whereas Dal Bello-Haas, et al. [24] reported blinding of the physical therapist performing the tests. The outcome evaluator was not blinded to group allocation in Drory, et al. [25]; however in Dal Bello-Haas, et al. [24] and Creemers, et al. [26] 2007, the evaluators were blinded to group allocation.

For non-RCT studies, the two cohort designs $[27,28]$ were identified as low and unclear risk of bias respectively and the cross-sectional study [29] was low risk of bias. This was concluded based on participants, outcomes, comparing baseline characteristics, and detection of reporting and/or attrition biases (Table 3).

\section{Discussion}

Currently there is little evidence quantifying positive and sustained effects of interventions from PT, OT and SLP on QoL in the ALS population. Across 5 studies, exercise and pain interventions by physiotherapists, communication devices and speech therapy and assistive aids have shown potential to impact functional outcomes in the management of people with ALS. However, only one study reported a statistically significant improvement in QoL, measured by the SF-36 [24]. The summary of quality of reporting the evidence (Table 2) suggests a reasonable quality as most studies were above $70 \%$. Additionally, the assessed risk of bias from the included studies were mainly low [24,26-28] or unclear [25,29], presenting a high quality of evidence overall. Nevertheless, the quantity is limited. 
Drory, et al. [25] and Dal Bello-Haas, et al. [24] have both demonstrated that exercise, when appropriately prescribed and performed, can positively affect physical function which may in turn impact QoL, particularly when individuals are still in the early stages of the disease. In fact, Dal Bello-Haas, et al. [24] found QoL to be significantly higher in the exercise group at 6 months, compared to a control group who completed usual activities. Even though exercise does not have the ability to improve strength of the muscles weakened by the condition, moderate regular exercises may have positive effects and can play an important part in the disease management [24]. The lack of strong evidence regarding the effectiveness and benefits of exercise on QoL in the ALS population may influence the availability and quality of rehabilitation interventions provided [4] and thus suggests a need for more research in this area.

Our included studies focused on interventions and/ or the influence of PT and SLP in managing people with ALS [24-27,29], rather than QoL. These have demonstrated that PT improves individual muscle strength and decreases fatigue and spasticity $[25,26]$. Augmentative and Alternative Communication (AAC) devices are shown to be helpful among individuals with ALS as there is an emotional aspect associated with communication that can affect one's QoL [9]. Although there is some overlap in the interventions provided by professionals from PT, OT, and SLP, the studies performed to date do not specifically address the impact of interventions by OTs in people with ALS. For example, assistive aids and appliances are often used in the management of ALS and can be recommended by both PTs and OTs (e.g., walkers, seating and positioning techniques, wheelchairs etc. [9]). Moreover, OT interventions can also be helpful for individuals with ALS in regards to employment, as engaging in activities that are essential to one's health and well-being. Yet, there is a lack of evidence establishing the impact such rehabilitation interventions have on a person's QoL, suggesting a need for further research.

Based on the results of this review, we identified few studies from the PT, OT and SLP professions that measured QoL outcomes of therapy interventions for people with ALS. We believe this may partly be due to the nature of ALS e.g., heterogeneity and rate of disease progression in the ALS population. Researchers who have performed RCTs in this area have consistently faced the challenge of high drop-out rates due to the quick progression of the disease $[25,26,29]$. Furthering ALS research through a longitudinal cohort study to examine which rehabilitation interventions have the potential to impact QoL would be beneficial. The literature available regarding the effectiveness of rehabilitation interventions is limited; however, there is growing evidence supporting the importance of multidisciplinary care for the management of ALS.

The strengths of this systematic review include a defined process and structured critical appraisal. The possibility of rejecting relevant studies was reduced by having two authors review all studies for inclusion using criteria agreed upon prior to extracting the data and reporting the quality of included studies. The quality of reporting the studies was assessed using the CONSORT Checklist [18] and STROBE Statement [20], and risk of bias using the Cochrane Risk of bias tool [19] and Newcastle-Ottawa Scale [21], thus allowing simple comparison of the results to identify study quality. Additionally, this study adds to the evidence by appraising the available literature to date and underlining the potential of rehabilitation services from PT, OT and SLP to maintain QoL when managed by a multidisciplinary team of specialists in rehabilitation.

\section{Limitations}

Research in ALS related specifically to PT, OT and SLP interventions is limited, and thus this systematic review is limited to a small number of studies, which had to be analysed descriptively. In addition, bias may have been introduced by restricting the review to only studies published in English.

\section{Conclusion}

The overall finding of this narrative review supports interventions from the professions of PT, OT and SLP have the potential to impact QoL in people with ALS, although the evidence demonstrating this effect, measured by the SF-36 and ALSAQ-40 is limited. It is difficult to determine the relative effects of rehabilitation interventions due to the disease progression in ALS, especially when the rate of progression differs across individuals, and evidence associated with rehabilitation and stage of ALS is very limited. Rehabilitation interventions can affect QoL and offering interventions that cater to the needs of people with ALS could contribute to the maintenance of QoL. Future research should be directed toward developing an approach to measure the effectiveness of different rehabilitation interventions in people with ALS and evaluating short and long-term effects of impairments on QoL.

\section{References}

1. ALS Therapy Development Institute (2015) ALS Frequently Asked Questions.

2. Francis K, Bach J, DeLisa J (1999) Evaluation and rehabilitation of patients with adult motor neuron disease. Arch Phys Med Rehabil 80: 951-963.

3. Blasco $\mathrm{H}$, Guennoc AM, Veyrat-Durebex C, Gordon $\mathrm{PH}$, Andres CR, et al. (2012) Amyotrophic lateral sclerosis: A hormonal condition? Amyotrophic Lateral Scler 13: 585588.

4. Forshew DA, Bromberg MB (2003) A survey of clinicians' practice in the symptomatic treatment of ALS. Amyotroph Lateral Scler Other Motor Neuron Disord 4: 258-263.

5. Kasarskis E (2004) Disease-modifying interventions. Amyotroph Lateral Scler Other Motor Neuron Disord 5: 76-78. 
6. Morris ME, Perry A, Bilney B, Curran A, Dodd K, et al (2006) Outcomes of physical therapy, speech pathology, and occupational therapy for people with motor neuron disease: A systematic review. Neurorehabil Neural Repair 20: 424-434.

7. Lui AJ, Byl NN (2009) A systematic review of the effect of moderate intensity exercise on function and disease progression in amyotrophic lateral sclerosis. J Neurol Phys Ther 33: 68-87.

8. Bromberg M, Forshew D (2001) Motor Neuron Disease. In: Pourmand R, Neuromuscular Diseases. ( $1^{\text {st }}$ edn), Butterworth Heinemann, Boston, USA, 67-103.

9. Ng L, Khan F, Mathers S (2009) Multidisciplinary care for adults with amyotrophic lateral sclerosis or motor neuron disease. Cochrane Database Syst Rev.

10. Oliver D (1996) The quality of care and symptom control the effects on the terminal phase of ALS/MND. J Neurol Sci 139: $134-136$.

11. Mitsumoto H, Bene $M(2000)$ Improving the quality of life for people with ALS: The challenge ahead. Amyotroph Lateral Scler Other Motor Neuron Disord 1: 329-336.

12. World Health Organization (WHO) (2005). Disability, including prevention, management and rehabilitation. Fifty-Eighth World Health Assembly.

13. Turner-Stokes L, Sykes N, Silber E (2008) Long-term neurological conditions: Management at the interface between neurology, rehabilitation and palliative care. Clin Med 8: 186-191.

14. Mikulic MA, DeLisa JA, Miller RM (1979) Rehabilitate the Patient with ALS? ARN J 4: 4-7.

15. Ware J, Snow K, Kosinski M, Gandek B (1993) SF-36 Health Survey: Manual and Interpretation Guide. The Health Institute, New England Medical Center, Boston, Massachusetts, USA.

16. Jenkinson C, Fitzpatrick R, Brennan C, Swash M (1999) Evidence for the validity and reliability of the ALS assessment questionnaire: The ALSAQ-40. Amyotrophic Lateral Scler Other Motor Neuron Disord 1: 33-40.

17. Feinstein AR, Cicchetti DV (1990) High agreement but low kappa: I. The problems of two paradoxes. J Clin Epidemiol 43: 543-549.

18. Schulz K, Altman DG, Moher D, CONSORT Group (2010) CONSORT 2010 Statement: Updated guidelines for reporting parallel group randomised trials. Trials 11: 32 .
19. Higgins J, Altman D, Gotzsche $P$, Juni $P$, Moher D, et al. (2011) The Cochrane Collaboration's tool for assessing risk of bias in randomised trials. BMJ 343: 5928.

20. Von Elm E, Altman DG, Egger M, Pocock SJ, Gøtzsche PC, et al. (2007) The Strengthening the Reporting of Observational Studies in Epidemiology (STROBE) statement: Guidelines for reporting observational studies. Epidemiology 18: $800-804$.

21. GA Wells, B Shea, D O'Connell, J Peterson, V Welch, et al. (2015) The Newcastle-Ottawa Scale (NOS) for assessing the quality of nonrandomised studies in meta-analyses.

22. Higgins JPT, Altman DG, Sterne JAC (2011) Assessing risk of bias in included studies. In: Higgins JPT, Green S Cochrane Handbook for Systematic Reviews of Interventions. The Cochrane Collaboration.

23. Dal Bello-Haas VD, Kloos A, Florence J, Scheirbecker J, Lopate G, et al. (2001) Effects of a strengthening program on Maximum Voluntary Isometric Contraction (MVIC) functional abilities, fatigue and Quality of Life (QoL) in individuals with Amyotrophic Lateral Sclerosis (ALS) - a preliminary study. Neurology Report 25: 127-128.

24. Dal Bello-Haas V, Florence JM, Kloos AD, Scheirbecker J, Lopate G, et al. (2007) A randomized controlled trial of resistance exercise in individuals with ALS. Neurology 68: 2003-2007.

25. Drory VE, Goltsman E, Reznik JG, Mosek A, Korczyn AD (2001) The value of muscle exercise in patients with amyotrophic lateral sclerosis. J Neurol Sci 191: 133-137.

26. Creemers H, Veldink JH, Grupstra H, Nollet F, Beelen A, et al. (2014) Cluster RCT of case management on patients' quality of life and caregiver strain in ALS. Neurology 82: 23-31.

27. Korner S, Siniawski M, Kollewe K, Rath KJ, Krampfl K, et al. (2012) Speech therapy and communication device: Impact on quality of life and mood in patients with amyotrophic lateral sclerosis. Amyotrophic Lateral Scler Frontotemporal Degener 14: 20-25.

28. Jensen MP, Abresch RT, Carter GT, McDonald CM (2005) Chronic Pain in Persons With Neuromuscular Disease. Arch Phys Med Rehabil 86: 1155-1163.

29. Van den Berg JP, Kalmijn S, Lindeman E, Veldink JH, de Visser M, et al. (2005) Multidisciplinary ALS care improves quality of life in patients with ALS. Neurology 65: 1264-1267. 